\title{
Iterative algorithms for finding the zeroes of sums of operators
}

\author{
Luo Yi Shi ${ }^{1}$, Rudong Chen ${ }^{1 *}$ and Yujing $\mathrm{Wu}^{2}$
}

"Correspondence:

chenrd@tjpu.edu.cn

${ }^{1}$ Department of Mathematics, Tianjin Polytechnic University,

Tianjin, 300387, P.R. China

Full list of author information is

available at the end of the article

\begin{abstract}
Let $H_{1}, H_{2}$ be real Hilbert spaces, $C \subseteq H_{1}$ be a nonempty closed convex set, and $0 \notin C$. Let $A: H_{1} \rightarrow H_{2}, B: H_{1} \rightarrow H_{2}$ be two bounded linear operators. We consider the problem to find $x \in C$ such that $A x=-B x(0=A x+B x)$. Recently, Eckstein and Svaiter presented some splitting methods for finding a zero of the sum of monotone operator $A$ and $B$. However, the algorithms are largely dependent on the maximal monotonicity of $A$ and $B$. In this paper, we describe some algorithms for finding a zero of the sum of $A$ and $B$ which ignore the conditions of the maximal monotonicity of $A$ and $B$.
\end{abstract}

Keywords: split equality problem; iterative algorithms; converge strongly

\section{Introduction and preliminaries}

Let $H_{1}, H_{2}, H_{3}$ be real Hilbert spaces, $C \subseteq H_{1}$ be a nonempty closed convex set and $0 \notin C$. Let $A: H_{1} \rightarrow H_{2}, B: H_{1} \rightarrow H_{2}$ be two bounded linear operators. We consider the interesting problem of finding $x \in C$ such that

$$
A x=-B x \quad(\text { or } 0=A x+B x) .
$$

For convenience, we denote the problem by $\mathcal{P}$.

For $\mathcal{P}$ it is generally difficult to find zeroes of $A$ and $B$ separately. To overcome this difficulty, Eckstein and Svaiter [1] present the splitting methods for finding a zero of the sum of monotone operator $A$ and $B$. Three basic families of splitting methods for this problem were identified in [1]:

(i) The Douglas/Peaceman-Rachford family, whose iteration is given by

$$
\begin{aligned}
& y_{k}=\left[2(I+\xi B)^{-1}+I\right] x_{k}, \\
& z_{k}=\left[2(I+\xi A)^{-1}+I\right] y_{k}, \\
& x_{k+1}=\left(1-\rho_{k}\right) x_{k}+\rho_{k} z_{k},
\end{aligned}
$$

where $\xi>0$ is a fixed scalar, and $\left\{\rho_{k}\right\} \subseteq(0,1]$ is a sequence of relaxation parameters.

(ii) The double backward splitting method, with iteration given by

$$
y_{k}=\left(I+\lambda_{k} B\right)^{-1} x_{k}
$$

O2014 Shi et al:; licensee Springer. This is an Open Access article distributed under the terms of the Creative Commons Attribution License (http://creativecommons.org/licenses/by/2.0), which permits unrestricted use, distribution, and reproduction in any medium, provided the original work is properly cited. 


$$
x_{k+1}=\left(I+\lambda_{k} A\right)^{-1} y_{k}
$$

where $\left\{\lambda_{k}\right\} \subseteq(0, \infty)$ a sequence of regularization parameters.

(iii) The forward-backward splitting method, with iteration given by

$$
\begin{aligned}
& y_{k} \in\left(I-\lambda_{k} A\right)^{-1} x_{k}, \\
& x_{k+1}=\left(I+\lambda_{k} B\right)^{-1} y_{k},
\end{aligned}
$$

where $\left\{\lambda_{k}\right\} \subseteq(0, \infty)$ a sequence of regularization parameters.

Convergence results for the scheme (i), in the case in which $\left\{\rho_{k}\right\}$ is contained in a compact subset of $(0,1)$, can be found in [2]; the convergence analysis of the double backward scheme given by (ii), which can be found in [3] and [4]; the standard convergence analysis for (iii) one can see [5]. However, the convergence results are largely dependent on the maximal monotonicity of $A$ and $B$. It is therefore the aim of this paper to construct new algorithms for problem $\mathcal{P}$ which ignore the conditions of the maximal monotonicity of $A$ and $B$.

The paper is organized as follows. In Section 2, we define the concept of the minimal norm solution of the problem $\mathcal{P}$ (1.1). Using Tychonov regularization, we obtain a net of solutions for some minimization problem approximating such minimal norm solution (see Theorem 2.4). In Section 3, we introduce an algorithm and prove the strong convergence of the algorithm, more importantly, its limit is the minimum-norm solution of the problem $\mathcal{P}$ (1.1) (see Theorem 3.2). In Section 4, we introduce KM-CQ-like iterative algorithm which converge strongly to a solution of the problem $\mathcal{P}(1.1)$ (see Theorem 4.3).

Throughout the rest of this paper, $I$ denotes the identity operator on Hilbert space $H$, $\operatorname{Fix}(T)$ the set of the fixed points of an operator $T$ and $\nabla f$ the gradient of the functional $f: H \rightarrow R$. An operator $T$ on a Hilbert space $H$ is nonexpansive if, for each $x$ and $y$ in $H$, $\|T x-T y\| \leq\|x-y\| . T$ is said to be averaged, if there exist $0<\alpha<1$ and a nonexpansive operator $N$ such that $T=(1-\alpha) I+\alpha N$.

We know that the projection $P_{C}$ from $H$ onto a nonempty closed convex subset $C$ of $H$ is a typical example of a nonexpansive and averaged mapping, which is defined by

$$
P_{C}(w)=\arg \min _{x \in C}\|x-w\| .
$$

It is well known that $P_{C}(w)$ is characterized by the inequality

$$
\left\langle w-P_{C}(w), x-P_{C}(w\rangle \leq 0, \quad \forall x \in C .\right.
$$

We now collect some elementary facts which will be used in the proofs of our main results.

Lemma 1.1 [6,7] Let $X$ be a Banach space, $C$ a closed convex subset of $X$, and $T: C \rightarrow C$ a nonexpansive mapping with $\operatorname{Fix}(T) \neq \emptyset$. If $\left\{x_{n}\right\}$ is a sequence in $C$ weakly converging to $x$ and if $\left\{(I-T) x_{n}\right\}$ converges strongly to $y$, then $(I-T) x=y$.

Lemma 1.2 [8] Let $\left\{s_{n}\right\}$ be a sequence of nonnegative real numbers, $\left\{\alpha_{n}\right\}$ a sequence of real numbers in $[0,1]$ with $\sum_{n=1}^{\infty} \alpha_{n}=\infty,\left\{u_{n}\right\}$ a sequence of nonnegative real numbers with 
$\sum_{n=1}^{\infty} u_{n}<\infty$, and $\left\{t_{n}\right\}$ a sequence of real numbers with lim $\sup _{n} t_{n} \leq 0$. Suppose that

$$
s_{n+1}=\left(1-\alpha_{n}\right) s_{n}+\alpha_{n} t_{n}+u_{n}, \quad \forall n \in N \text {. }
$$

Then $\lim _{n \rightarrow \infty} s_{n}=0$.

Lemma 1.3 [9] Let $\left\{w_{n}\right\},\left\{z_{n}\right\}$ be bounded sequences in a Banach space and let $\left\{\beta_{n}\right\}$ be a sequence in $[0,1]$ which satisfies the following condition: $0<\liminf _{n \rightarrow \infty} \beta_{n} \leq \limsup _{n \rightarrow \infty} \beta_{n}<$ 1. Suppose that $w_{n+1}=\left(1-\beta_{n}\right) w_{n}+\beta_{n} z_{n}$ and $\lim \sup _{n \rightarrow \infty}\left\|z_{n+1}-z_{n}\right\|-\left\|w_{n+1}-w_{n}\right\| \leq 0$, then $\lim _{n \rightarrow \infty}\left\|z_{n}-w_{n}\right\|=0$.

Lemma 1.4 [10] Let $f$ be a convex and differentiable functional and let $C$ be a closed convex subset of $H$. Then $x \in C$ is a solution of the problem

$$
\min _{x \in C} f(x)
$$

if and only if $x \in C$ satisfies the following optimality condition:

$$
\langle\nabla f(x), v-x\rangle \geq 0, \quad \forall v \in C
$$

Moreover, iff is, in addition, strictly convex and coercive, then the minimization problem has a unique solution.

Lemma 1.5 [11] Let $A$ and $B$ be averaged operators and suppose that $\operatorname{Fix}(A) \cap \operatorname{Fix}(B)$ is nonempty. Then $\operatorname{Fix}(A) \cap \operatorname{Fix}(B)=\operatorname{Fix}(A B)=\operatorname{Fix}(B A)$.

\section{The minimum-norm solution of the problem $\mathcal{P}$}

In this section, we propose the concept of the minimal norm solution of $\mathcal{P}$ (1.1). Then, using Tychonov regularization, we obtain the minimal norm solution by a net of solution for some minimization problem.

We use $\Gamma$ to denote the solution set of $\mathcal{P}$, i.e.,

$$
\Gamma=\left\{x \in H_{1}, A x=-B x, x \in C\right\}
$$

and assume consistency of $\mathcal{P}$. Hence $\Gamma$ is closed, convex, and nonempty.

Let $H=H_{1} \times H_{1}, M=\left\{(x, x), x \in H_{1}\right\} \subseteq H, P$ be the linear operator from $H_{1}$ onto $M$, and $P$ has the matrix form

$$
P=\left[\begin{array}{l}
I \\
I
\end{array}\right],
$$

that is to say, $P(x)=(x, x), \forall x \in H_{1}$.

Define $G: H \rightarrow H_{2}$ by $G((x, y))=A x+B y, \forall(x, y) \in H_{2}$. Then $G$ has the matrix form $G=[A, B]$, and $G P=A+B, P G^{*} G P=A^{*} A+A^{*} B+B^{*} A+B^{*} B$.

The problem can now be reformulated as finding $x \in C$ with $G P x=0$, or solving the following minimization problem:

$$
\min _{x \in C} f(x)=\frac{1}{2}\|G P x\|^{2}
$$


which is ill-posed. A classical way is the well-known Tychonov regularization, which approximates a solution of problem (2.1) by the unique minimizer of the regularized problem:

$$
\min _{x \in C} f_{\alpha}(x)=\frac{1}{2}\|G P x\|^{2}+\frac{1}{2} \alpha\|x\|^{2}
$$

where $\alpha>0$ is the regularization parameter. Denote by $x_{\alpha}$ the unique solution of (2.2).

Proposition 2.1 For $\alpha>0$, the solution $x_{\alpha}$ of (2.2) is uniquely defined. $x_{\alpha}$ is characterized by the inequality

$$
\left\langle P^{*} G^{*} G P x_{\alpha}+\alpha x_{\alpha}, x-x_{\alpha}\right\rangle \geq 0, \quad \forall x \in C .
$$

Proof Obviously, $f(x)=\frac{1}{2}\|G P x\|^{2}$ is convex and differentiable with gradient $\nabla f(x)=$ $P^{*} G^{*} G P x$. Recall that $f_{\alpha}(x)=f(x)+\frac{1}{2} \alpha\|x\|^{2}$, we see that $f_{\alpha}$ is strictly convex and differentiable with gradient

$$
\nabla f_{\alpha}(x)=P^{*} G^{*} G P x+\alpha x
$$

According Lemma 1.4, $x_{\alpha}$ is characterized by the inequality

$$
\left\langle P^{*} G^{*} G P x_{\alpha}+\alpha x_{\alpha}, x-x_{\alpha}\right\rangle \geq 0, \quad \forall x \in C .
$$

Definition 2.2 An element $\tilde{x} \in \Gamma$ is said to be the minimal norm solution of SEP (1.1) if $\|\tilde{x}\|=\inf _{x \in \Gamma}\|x\|$.

The following proposition collects some useful properties of $\left\{x_{\alpha}\right\}$ the unique solution of (2.2).

Proposition 2.3 Let $x_{\alpha}$ be given as the unique solution of (2.2). Then we have:

(i) $\left\|x_{\alpha}\right\|$ is decreasing for $\alpha \in(0, \infty)$.

(ii) $\alpha \mapsto x_{\alpha}$ defines a continuous curve from $(0, \infty)$ to $H_{1}$.

Proof Let $\alpha>\beta>0$, since $x_{\alpha}$ and $x_{\beta}$ are the unique minimizers of $f_{\alpha}$ and $f_{\beta}$, respectively, we get

$$
\begin{aligned}
& \frac{1}{2}\left\|G P x_{\alpha}\right\|^{2}+\frac{1}{2} \alpha\left\|x_{\alpha}\right\|^{2} \leq \frac{1}{2}\left\|G P x_{\beta}\right\|^{2}+\frac{1}{2} \alpha\left\|x_{\beta}\right\|^{2}, \\
& \frac{1}{2}\left\|G P x_{\beta}\right\|^{2}+\frac{1}{2} \beta\left\|x_{\beta}\right\|^{2} \leq \frac{1}{2}\left\|G P x_{\alpha}\right\|^{2}+\frac{1}{2} \beta\left\|x_{\alpha}\right\|^{2} .
\end{aligned}
$$

It follows that $\left\|x_{\alpha}\right\| \leq\left\|x_{\beta}\right\|$. Thus $\left\|x_{\alpha}\right\|$ is decreasing for $\alpha \in(0, \infty)$.

According to Proposition 2.1, we get

$$
\left\langle P^{*} G^{*} G P x_{\alpha}+\alpha x_{\alpha}, x_{\beta}-x_{\alpha}\right\rangle \geq 0
$$


and

$$
\left\langle P^{*} G^{*} G P x_{\beta}+\beta x_{\beta}, x_{\alpha}-x_{\beta}\right\rangle \geq 0 .
$$

It follows that

$$
\left\langle x_{\alpha}-x_{\beta}, \alpha x_{\alpha}-\beta x_{\beta}\right\rangle \leq\left\langle x_{\alpha}-x_{\beta}, P^{*} G^{*} G P\left(x_{\beta}-x_{\alpha}\right)\right\rangle \leq 0 .
$$

Thus

$$
\alpha\left\|x_{\alpha}-x_{\beta}\right\| \leq(\alpha-\beta)\left\langle x_{\alpha}-x_{\beta}, x_{\beta}\right\rangle .
$$

It turns out that

$$
\left\|x_{\alpha}-x_{\beta}\right\|^{2} \leq \frac{|\alpha-\beta|}{\alpha}\left\|x_{\beta}\right\| .
$$

Hence, $\alpha \mapsto x_{\alpha}$ is a continuous curve from $(0, \infty)$ to $H_{1}$.

Theorem 2.4 Let $x_{\alpha}$ be the unique solution of (2.2). Then $x_{\alpha}$ converges strongly to the minimum-norm solution $\tilde{x}$ of $\mathcal{P}(1.1)$ with $\alpha \rightarrow 0$.

Proof For any $0<\alpha<\infty, x_{\alpha}$ is given as (2.2), we get

$$
\frac{1}{2}\left\|G P x_{\alpha}\right\|^{2}+\frac{1}{2} \alpha\left\|x_{\alpha}\right\|^{2} \leq \frac{1}{2}\|G P \tilde{x}\|^{2}+\frac{1}{2} \alpha\|\tilde{x}\|^{2} .
$$

Since $\tilde{x} \in \Gamma$ is a solution for $\mathcal{P}$,

$$
\frac{1}{2}\left\|G P x_{\alpha}\right\|^{2}+\frac{1}{2} \alpha\left\|x_{\alpha}\right\|^{2} \leq \frac{1}{2} \alpha\|\tilde{x}\|^{2} .
$$

It follows that $\left\|x_{\alpha}\right\| \leq\|\tilde{x}\|$ for all $\alpha>0$. Thus $\left\{x_{\alpha}\right\}$ is a bounded net in $H_{1}$.

All we need to prove is that for any sequence $\left\{\alpha_{n}\right\}$ such that $\alpha_{n} \rightarrow 0,\left\{x_{\alpha_{n}}\right\}$ contains a subsequence converging strongly to $\tilde{x}$. For convenience, we set $x_{n}=x_{\alpha_{n}}$.

In fact $\left\{x_{n}\right\}$ is bounded, by passing to a subsequence if necessary, we may assume that $\left\{x_{n}\right\}$ converges weakly to a point $\hat{x} \in S$. Due to Proposition 2.1, we get

$$
\left\langle P^{*} G^{*} G P x_{n}+\alpha_{n} x_{n}, \tilde{x}-x_{n}\right\rangle \geq 0 .
$$

It turns out that

$$
\left\langle G P x_{n}, G P \tilde{x}-G P x_{n}\right\rangle \geq \alpha_{n}\left\langle x_{n}, x_{n}-\tilde{x}\right\rangle .
$$

Since $\tilde{x} \in \Gamma$, it follows that

$$
\left\langle G P x_{n},-G P x_{n}\right\rangle \geq \alpha_{n}\left\langle x_{n}, x_{n}-\tilde{x}\right\rangle .
$$

Noting that $\left\|x_{n}\right\| \leq\|\tilde{x}\|$, we have

$$
\left\|G P x_{n}\right\| \leq 2 \alpha_{n}\|\tilde{x}\| \rightarrow 0 .
$$


Moreover, note that $\left\{x_{n}\right\}$ converges weakly to a point $\hat{x} \in C$, thus $\left\{G P x_{n}\right\}$ converges weakly to GP $\hat{x}$. It follows that $G P \hat{x}=0$, i.e. $\hat{x} \in \Gamma$.

Finally, we prove that $\hat{x}=\tilde{x}$ and this finishes the proof.

Recall that $\left\{x_{n}\right\}$ converges weakly to $\hat{x}$ and $\left\|x_{n}\right\| \leq\|\tilde{x}\|$, one can deduce that

$$
\|\hat{x}\| \leq \liminf _{n}\left\|x_{n}\right\| \leq\|\tilde{x}\|=\min \{\|x\|: x \in \Gamma\}
$$

This shows that $\hat{x}$ is also a point in $\Gamma$ with minimum-norm. By the uniqueness of minimum-norm element, we get $\hat{x}=\tilde{x}$.

Finally, we will introduce another method to get the minimum-norm solution of the problem $\mathcal{P}$.

Lemma 2.5 Let $T=I-\gamma P^{*} G^{*} G P$, where $0<\gamma<2 / \rho\left(P^{*} G^{*} G P\right)$ with $\rho\left(P^{*} G^{*} G P\right)$ being the spectral radius of the self-adjoint operator $P^{*} G^{*} G P$ on $H_{1}$. Then we have the following:

(1) $\|T\| \leq 1$ (i.e. $T$ is nonexpansive) and averaged;

(2) $\operatorname{Fix}(T)=\left\{x \in H_{1}, A x=-B x\right\}, \operatorname{Fix}\left(P_{C} T\right)=\operatorname{Fix}\left(P_{C}\right) \cap \operatorname{Fix}(T)=\Gamma$;

(3) $x \in \operatorname{Fix}\left(P_{C} T\right)$ if and only if $x$ is a solution of the variational inequality

$\left\langle P^{*} G^{*} G P x, v-x\right\rangle \geq 0, \forall v \in C$.

Proof (1) It is easily proved that $\|T\| \leq 1$, we only need to prove that $T=I-\gamma P^{*} G^{*} G P$ is averaged. Indeed, choose $0<\beta<1$, such that $\gamma /(1-\beta)<2 / \rho\left(P^{*} G^{*} G P\right)$, then $T=I-$ $\gamma P^{*} G^{*} G P=\beta I+(1-\beta) V$, where $V=I-\gamma /(1-\beta) P^{*} G^{*} G P$ is a nonexpansive mapping. That is to say $T$ is averaged.

(2) If $x \in\left\{x \in H_{1}, A x=-B x\right\}$, it is obviously that $x \in \operatorname{Fix}(T)$. Conversely, assume that $x \in$ Fix $(T)$, we have $x=x-\gamma P^{*} G^{*} G P x$, hence $\gamma P^{*} G^{*} G P x=0$ then $\|G P x\|^{2}=\left\langle P^{*} G^{*} G P x, x\right\rangle=0$, we get $x \in\left\{x \in H_{1}, A x=-B x\right\}$. We have $\operatorname{Fix}(T)=\left\{x \in H_{1}, A x=-B x\right\}$.

Now we prove $\operatorname{Fix}\left(P_{C} T\right)=\operatorname{Fix}\left(P_{C}\right) \cap \operatorname{Fix}(T)=\Gamma$. By $\operatorname{Fix}(T)=\left\{x \in H_{1}, A x=-B x\right\}$, $\operatorname{Fix}\left(P_{C}\right) \cap \operatorname{Fix}(T)=\Gamma$ is obviously. On the other hand, since $\operatorname{Fix}\left(P_{C}\right) \cap \operatorname{Fix}(T)=\Gamma \neq \emptyset$, and both $P_{C}$ and $T$ are averaged, from Lemma 1.5, we have $\operatorname{Fix}\left(P_{C} T\right)=\operatorname{Fix}\left(P_{C}\right) \cap \operatorname{Fix}(T)$.

(3)

$$
\begin{aligned}
\left\langle P^{*} G^{*} G P x, v-x\right\rangle \geq 0, \quad \forall v \in C & \Leftrightarrow\left\langle x-\left(x-\gamma P^{*} G^{*} G P x\right), v-x\right\rangle \geq 0, \quad \forall v \in S \\
& \Leftrightarrow w=P_{C}\left(w-\gamma P^{*} G^{*} G P x\right) \\
& \Leftrightarrow w \in \operatorname{Fix}\left(P_{C} T\right) .
\end{aligned}
$$

Remark 2.6 Choose a constant $\gamma$ satisfying that $0<\gamma<2 / \rho\left(P^{*} G^{*} G P\right)$. For $\alpha \in(0$, $\left.\frac{2-\gamma\left\|P^{*} G^{*} G P\right\|}{2 \gamma}\right)$, we define a mapping

$$
W_{\alpha}(x):=P_{C}\left[(1-\alpha \gamma) I-\gamma P^{*} G^{*} G P\right] x .
$$

It is clear that $W_{\alpha}$ is a contractive. Hence, $W_{\alpha}$ has a unique fixed point $x_{\alpha}$, we have

$$
x_{\alpha}=P_{C}\left[(1-\alpha \gamma) I-\gamma P^{*} G^{*} G P\right] x_{\alpha} .
$$

Theorem 2.7 Let $x_{\alpha}$ be given as (2.4). Then $x_{\alpha}$ converges strongly to the minimum-norm solution $\tilde{x}$ of the problem $\mathcal{P}$ (1.1) when $\alpha \rightarrow 0$. 
Proof Choose $\check{x} \in \Gamma$, noting that $\alpha \in\left(0, \frac{2-\gamma\left\|P^{*} G^{*} G P\right\|}{2 \gamma}\right), I-\frac{\gamma}{(1-\alpha \gamma)} P^{*} G^{*} G P$ is nonexpansive, it turns out that

$$
\begin{aligned}
\left\|x_{\alpha}-\check{x}\right\|= & \left\|P_{C}\left[(1-\alpha \gamma) I-\gamma P^{*} G^{*} G P\right] x_{\alpha}-P_{C}\left[\check{x}-\gamma P^{*} G^{*} G P \check{x}\right]\right\| \\
\leq & \left\|\left[(1-\alpha \gamma) I-\gamma P^{*} G^{*} G P\right] x_{\alpha}-\left[\check{x}-\gamma P^{*} G^{*} G P \check{x}\right]\right\| \\
= & \|(1-\alpha \gamma)\left[x_{\alpha}-\frac{\gamma}{1-\alpha \gamma} P^{*} G^{*} G P x_{\alpha}\right] \\
& -(1-\alpha \gamma)\left[\check{x}-\frac{\gamma}{1-\alpha \gamma} P^{*} G^{*} G P \check{x}\right]-\alpha \gamma \check{x} \| \\
\leq & (1-\alpha \gamma)\left\|\left(x_{\alpha}-\frac{\gamma}{1-\alpha \gamma} P^{*} G^{*} G P x_{\alpha}\right)-\left(\check{x}-\frac{\gamma}{1-\alpha \gamma} P^{*} G^{*} G P \check{x}\right)\right\|+\alpha \gamma\|\check{x}\| \\
\leq & (1-\alpha \gamma)\left\|x_{\alpha}-\check{x}\right\|+\alpha \gamma\|\check{x}\| .
\end{aligned}
$$

That is,

$$
\left\|x_{\alpha}-\check{x}\right\| \leq\|\check{x}\|
$$

Hence $\left\{x_{\alpha}\right\}$ is bounded.

Taking into account of (2.4), we have

$$
\left\|x_{\alpha}-P_{C}\left[I-\gamma P^{*} G^{*} G P\right] x_{\alpha}\right\| \leq \alpha\left\|\gamma x_{\alpha}\right\| \rightarrow 0
$$

We assert that $\left\{x_{\alpha}\right\}$ is relatively norm compact as $\alpha \rightarrow 0^{+}$. In fact, assume that $\left\{\alpha_{n}\right\} \subseteq$ $\left(0, \frac{2-\gamma\left\|P^{*} G^{*} G P\right\|}{2 \gamma}\right)$ and $\alpha_{n} \rightarrow 0^{+}$as $n \rightarrow \infty$. For convenience, we put $x_{n}:=x_{\alpha_{n}}$, we get

$$
\left\|x_{n}-P_{C}\left[I-\gamma P^{*} G^{*} G P\right] x_{n}\right\| \leq \alpha_{n}\left\|\gamma x_{n}\right\| \rightarrow 0 .
$$

Since $P_{C}$ is nonexpansive, one concludes that

$$
\begin{aligned}
\left\|x_{\alpha}-\check{x}\right\|^{2}= & \left\|P_{C}\left[(1-\alpha \gamma) I-\gamma P^{*} G^{*} G P\right] x_{\alpha}-P_{C}\left[\check{x}-\gamma P^{*} G^{*} G P \check{x}\right]\right\|^{2} \\
\leq & \left\langle\left[(1-\alpha \gamma) I-\gamma P^{*} G^{*} G P\right] x_{\alpha}-\left[\check{x}-\gamma P^{*} G^{*} G P \check{x}\right], x_{\alpha}-\check{x}\right\rangle \\
= & \left\langle(1-\alpha \gamma)\left[x_{\alpha}-\frac{\gamma}{1-\alpha \gamma} P^{*} G^{*} G P x_{\alpha}\right]\right. \\
& \left.-(1-\alpha \gamma)\left[\check{x}-\frac{\gamma}{1-\alpha \gamma} P^{*} G^{*} G P \check{x}\right], x_{\alpha}-\check{x}\right\rangle-\alpha \gamma\left\langle\check{x}, x_{\alpha}-\check{x}\right\rangle \\
\leq & (1-\alpha \gamma)\left\|x_{\alpha}-\check{x}\right\|^{2}-\alpha \gamma\left\langle\check{x}, x_{\alpha}-\check{x}\right\rangle .
\end{aligned}
$$

That is ,

$$
\left\|x_{\alpha}-\check{x}\right\|^{2} \leq\left\langle-\check{x}, x_{\alpha}-\check{x}\right\rangle .
$$

Thus,

$$
\left\|x_{n}-\check{x}\right\|^{2} \leq\left\langle-\check{x}, x_{n}-\check{x}\right\rangle, \quad \forall \check{x} \in \Gamma .
$$


Due to $\left\{x_{n}\right\}$ is bounded, there exists a subsequence of $\left\{x_{n}\right\}$ which converges weakly to a point $\tilde{x}$. Without loss of generality, we may assume that $\left\{x_{n}\right\}$ converges weakly to $\tilde{x}$. Noting that

$$
\left\|x_{n}-P_{C}\left[I-\gamma P^{*} G^{*} G P\right] x_{n}\right\| \leq \alpha_{n}\left\|\gamma x_{n}\right\| \rightarrow 0,
$$

and applying Lemma 1.1, we obtain $\tilde{x} \in \operatorname{Fix}\left(P_{C}\left[I-\gamma P^{*} G^{*} G P\right]\right)=\Gamma$.

Since

$$
\left\|x_{n}-\check{x}\right\|^{2} \leq\left\langle-\check{x}, x_{n}-\check{x}\right\rangle, \quad \forall \check{x} \in \Gamma,
$$

it concludes that

$$
\left\|x_{n}-\tilde{x}\right\|^{2} \leq\left\langle-\tilde{x}, x_{n}-\tilde{x}\right\rangle
$$

Hence, if $\left\{x_{n}\right\}$ converges weakly to $\tilde{x}$, then $\left\{x_{n}\right\}$ converges strongly to $\tilde{x}$. That is to say $\left\{x_{\alpha}\right\}$ is relatively norm compact as $\alpha \rightarrow 0^{+}$.

Moreover, again using

$$
\left\|x_{n}-\check{x}\right\|^{2} \leq\left\langle-\check{x}, x_{n}-\check{x}\right\rangle, \quad \forall \check{x} \in \Gamma,
$$

let $n \rightarrow \infty$, we have

$$
\|\tilde{x}-\check{x}\|^{2} \leq\langle-\check{x}, \tilde{x}-\check{x}\rangle, \quad \forall \check{x} \in \Gamma .
$$

This implies that

$$
\langle-\check{x}, \check{x}-\tilde{x}\rangle \leq 0, \quad \forall \check{x} \in \Gamma .
$$

This is equivalent to

$$
\langle-\tilde{x}, \check{x}-\tilde{x}\rangle \leq 0, \quad \forall \check{x} \in \Gamma .
$$

It turns out that $\tilde{x} \in P_{C}(0)$. Consequently, each cluster point of $x_{\alpha}$ is equals $\tilde{x}$. Thus $x_{\alpha} \rightarrow$ $\tilde{x}(\alpha \rightarrow 0)$ the minimum-norm solution of the problem $\mathcal{P}$.

\section{Iterative algorithm for the minimum-norm solution of the problem $\mathcal{P}$}

In this section, we introduce the following algorithm and prove the strong convergence of the algorithm, more importantly, its limit is the minimum-norm solution of the problem $\mathcal{P}$.

Algorithm 3.1 For an arbitrary point $x_{0} \in H_{1}$ the sequence $\left\{x_{n}\right\}$ is generated by the iterative algorithm

$$
x_{n+1}=P_{C}\left\{\left(1-\alpha_{n}\right)\left[I-\gamma P^{*} G^{*} G P\right] x_{n}\right\},
$$

where $\alpha_{n}>0$ is a sequence in $(0,1)$ such that 
(i) $\lim _{n} \alpha_{n}=0$;

(ii) $\sum_{n=0}^{\infty} \alpha_{n}=\infty$;

(iii) $\sum_{n=0}^{\infty}\left|\alpha_{n+1}-\alpha_{n}\right|<\infty$ or $\lim _{n}\left|\alpha_{n+1}-\alpha_{n}\right| / \alpha_{n}=0$.

Now, we prove the strong convergence of the iterative algorithm.

Theorem 3.2 The sequence $\left\{x_{n}\right\}$ generated by algorithm (3.1) converges strongly to the minimum-norm solution $\tilde{x}$ of the problem $\mathcal{P}(1.1)$.

Proof Let $R_{n}$ and $R$ be defined by

$$
\begin{aligned}
& R_{n} x:=P_{C}\left\{\left(1-\alpha_{n}\right)\left[I-\gamma P^{*} G^{*} G P\right]\right\} x=P_{C}\left[\left(1-\alpha_{n}\right) T x\right], \\
& R x:=P_{C}\left(I-\gamma P^{*} G^{*} G P\right) x=P_{C}(T x),
\end{aligned}
$$

where $T=I-\gamma P^{*} G^{*} G P$, by Lemma 2.5 , it is easy to see that $R_{n}$ is a contraction with contractive constant $1-\alpha_{n}$. Algorithm (3.1) can be written as $x_{n+1}=R_{n} x_{n}$.

For any $\hat{x} \in \Gamma$, we have

$$
\begin{aligned}
\left\|R_{n} \hat{x}-\hat{x}\right\| & =\left\|P_{C}\left[\left(1-\alpha_{n}\right) T \hat{x}\right]-\hat{x}\right\| \\
& =\left\|P_{C}\left[\left(1-\alpha_{n}\right) T \hat{x}\right]-P_{S}(T \hat{x})\right\| \\
& \leq\left\|\left(1-\alpha_{n}\right) T \hat{x}-T \hat{x}\right\| \\
& =\alpha_{n}\|T \hat{x}\| \leq \alpha_{n}\|\hat{x}\| .
\end{aligned}
$$

Hence,

$$
\begin{aligned}
\left\|x_{n+1}-\hat{x}\right\| & =\left\|R_{n} x_{n}-\hat{x}\right\| \leq\left\|R_{n} x_{n}-R_{n} \hat{x}\right\|+\left\|R_{n} \hat{x}-\hat{x}\right\| \\
& \leq\left\|P_{C}\left[\left(1-\alpha_{n}\right) T \hat{x}\right]-P_{S}(T \hat{x})\right\| \\
& \leq\left(1-\alpha_{n}\right)\left\|x_{n}-\hat{x}\right\|+\alpha_{n}\|\hat{x}\| \\
& \leq \max \left\{\left\|x_{n}-\hat{x}\right\|,\|\hat{x}\|\right\} .
\end{aligned}
$$

It follows that $\left\|x_{n}-\hat{x}\right\| \leq \max \left\{\left\|x_{0}-\hat{x}\right\|,\|\hat{x}\|\right\}$. So $\left\{x_{n}\right\}$ is bounded.

Next we prove that $\lim _{n}\left\|x_{n+1}-x_{n}\right\|=0$.

Indeed,

$$
\begin{aligned}
\left\|x_{n+1}-x_{n}\right\| & =\left\|R_{n} x_{n}-R_{n-1} x_{n-1}\right\| \\
& \leq\left\|R_{n} x_{n}-R_{n} x_{n-1}\right\|+\left\|R_{n} x_{n-1}-R_{n-1} x_{n-1}\right\| \\
& \leq\left(1-\alpha_{n}\right)\left\|x_{n}-x_{n-1}\right\|+\left\|R_{n} x_{n-1}-R_{n-1} x_{n-1}\right\| .
\end{aligned}
$$

Notice that

$$
\begin{aligned}
\left\|R_{n} x_{n-1}-R_{n-1} x_{n-1}\right\| & =\left\|P_{C}\left[\left(1-\alpha_{n}\right) T x_{n-1}\right]-P_{C}\left[\left(1-\alpha_{n-1}\right) T x_{n-1}\right]\right\| \\
& \leq\left\|\left(1-\alpha_{n}\right) T x_{n-1}-\left(1-\alpha_{n-1}\right) T x_{n-1}\right\|
\end{aligned}
$$




$$
\begin{aligned}
& =\left|\alpha_{n}-\alpha_{n-1}\right|\left\|T x_{n-1}\right\| \\
& \leq\left|\alpha_{n}-\alpha_{n-1}\right|\left\|x_{n-1}\right\| .
\end{aligned}
$$

Hence

$$
\left\|x_{n+1}-x_{n}\right\| \leq\left(1-\alpha_{n}\right)\left\|x_{n}-x_{n-1}\right\|+\left|\alpha_{n}-\alpha_{n-1}\right|\left\|x_{n-1}\right\| .
$$

By virtue of the assumptions (1)-(3) and Lemma 1.2, we have

$$
\lim _{n}\left\|x_{n+1}-x_{n}\right\|=0
$$

Therefore,

$$
\begin{aligned}
\left\|x_{n}-R x_{n}\right\| & \leq\left\|x_{n+1}-x_{n}\right\|+\left\|R_{n} x_{n}-R x_{n}\right\| \\
& \leq\left\|x_{n+1}-x_{n}\right\|+\left\|\left(1-\alpha_{n}\right) T x_{n}-T x_{n}\right\| \\
& \leq\left\|x_{n+1}-x_{n}\right\|+\alpha_{n}\left\|x_{n}\right\| \rightarrow 0 .
\end{aligned}
$$

By the demiclosedness principle ensures that each weak limit point of $\left\{x_{n}\right\}$ is a fixed point of the nonexpansive mapping $R=P_{C} T$, that is, a point of the solution set $\Gamma$ of SEP (1.1).

Finally, we will prove that $\lim _{n}\left\|x_{n+1}-\tilde{x}\right\|=0$.

Choose $0<\beta<1$, such that $\gamma /(1-\beta)<2 / \rho\left(P^{*} G^{*} G P\right)$, then $T=I-\gamma P^{*} G^{*} G P=\beta I+(1-$ $\beta) V$, where $V=I-\gamma /(1-\beta) P^{*} G^{*} G P$ is a nonexpansive mapping. Taking $z \in \Gamma$, we deduce that

$$
\begin{aligned}
\left\|x_{n+1}-z\right\|^{2} & =\left\|P_{C}\left[\left(1-\alpha_{n}\right) T x_{n}\right]-z\right\|^{2} \\
& \leq\left\|\left(1-\alpha_{n}\right) T x_{n}-z\right\|^{2} \\
& \leq\left(1-\alpha_{n}\right)\left\|T x_{n}-z\right\|^{2}+\alpha_{n}\|z\|^{2} \\
& \leq\left\|\beta\left(x_{n}-z\right)+(1-\beta)\left(V x_{n}-z\right)\right\|^{2}+\alpha_{n}\|z\|^{2} \\
& \leq \beta\left\|\left(x_{n}-z\right)\right\|^{2}+(1-\beta)\left\|\left(V x_{n}-z\right)\right\|^{2}-\beta(1-\beta)\left\|x_{n}-V x_{n}\right\|^{2}+\alpha_{n}\|z\|^{2} \\
& \leq\left\|\left(x_{n}-z\right)\right\|^{2}-\beta(1-\beta)\left\|x_{n}-V x_{n}\right\|^{2}+\alpha_{n}\|z\|^{2} .
\end{aligned}
$$

Then

$$
\begin{aligned}
\beta(1-\beta)\left\|x_{n}-V x_{n}\right\| & \leq\left\|x_{n}-z\right\|^{2}-\left\|x_{n+1}-z\right\|^{2}+\alpha_{n}\|z\|^{2} \\
& \leq\left(\left\|x_{n}-z\right\|+\left\|x_{n+1}-z\right\|\right)\left(\left\|x_{n}-z\right\|-\left\|x_{n+1}-z\right\|\right) \alpha_{n}\|z\|^{2} \\
& \leq\left(\left\|x_{n}-z\right\|+\left\|x_{n+1}-z\right\|\right)\left(\left\|x_{n}-x_{n+1}\right\|\right) \alpha_{n}\|z\|^{2} \rightarrow 0 .
\end{aligned}
$$

Note that $T=I-\gamma P^{*} G^{*} G P=\beta I+(1-\beta) V$, it follows that $\lim _{n}\left\|T x_{n}-x_{n}\right\|=0$.

Take a subsequence $\left\{x_{n_{k}}\right\}$ of $\left\{x_{n}\right\}$ such that $\limsup _{n}\left\langle x_{n}-\tilde{x},-\tilde{x}\right\rangle=\lim _{k}\left\langle x_{n_{k}}-\tilde{x},-\tilde{x}\right\rangle$.

By virtue of the boundedness of $x_{n}$, we may further assume with no loss of generality that $x_{n_{k}}$ converges weakly to a point $\check{x}$. Since $\left\|R x_{n}-x_{n}\right\| \rightarrow 0$, using the demiclosedness 
principle, $\check{x} \in \operatorname{Fix}(R)=\operatorname{Fix}\left(P_{C} T\right)=\Gamma$. Noticing that $\tilde{x}$ is the projection of the origin onto $\Gamma$, we get

$$
\limsup _{n}\left\langle x_{n}-\tilde{x},-\tilde{x}\right\rangle=\lim _{k}\left\langle x_{n_{k}}-\tilde{x},-\tilde{x}\right\rangle=\langle\check{x}-\tilde{x},-\tilde{x}\rangle \leq 0 .
$$

Finally, we compute

$$
\begin{aligned}
\left\|x_{n+1}-\tilde{x}\right\|^{2} & =\left\|P_{C}\left[\left(1-\alpha_{n}\right) T x_{n}\right]-\tilde{x}\right\|^{2} \\
& =\left\|P_{C}\left[\left(1-\alpha_{n}\right) T x_{n}\right]-P_{C} T \tilde{x}\right\|^{2} \\
& \leq\left\|\left(1-\alpha_{n}\right) T x_{n}-T \tilde{x}\right\|^{2} \\
& =\left\|\left(1-\alpha_{n}\right) T x_{n}-\tilde{x}\right\|^{2} \\
& =\left\|\left(1-\alpha_{n}\right)\left(T x_{n}-\tilde{x}\right)+\alpha_{n}(-\tilde{x})\right\|^{2} \\
& =\left(1-\alpha_{n}\right)^{2}\left\|\left(T x_{n}-\tilde{x}\right)\right\|^{2}+\alpha_{n}^{2}\|\tilde{x}\|^{2}+2 \alpha_{n}\left(1-\alpha_{n}\right)\left\langle T x_{n}-\tilde{x},-\tilde{x}\right\rangle \\
& \leq\left(1-\alpha_{n}\right)\left\|\left(T x_{n}-\tilde{x}\right)\right\|^{2}+\alpha_{n}\left[\alpha_{n}\|\tilde{x}\|^{2}+2\left(1-\alpha_{n}\right)\left\langle T x_{n}-\tilde{x},-\tilde{x}\right\rangle\right] .
\end{aligned}
$$

Since, $\lim \sup _{n}\left\langle x_{n}-\tilde{x},-\tilde{x}\right\rangle \leq 0,\left\|x_{n}-T x_{n}\right\| \rightarrow 0$, we know that $\limsup _{n}\left(\alpha_{n}\|\tilde{x}\|^{2}+2(1-\right.$ $\left.\left.\alpha_{n}\right)\left\langle T x_{n}-\tilde{x},-\tilde{x}\right\rangle\right) \leq 0$, by Lemma 1.2, we conclude that $\lim _{n}\left\|x_{n+1}-\tilde{x}\right\|=0$. This completes the proof.

\section{$4 \mathrm{KM}$-CQ-like iterative algorithm for the problem $\mathcal{P}$}

In this section, we establish a KM-CQ-like algorithm converges strongly to a solution of the problem $\mathcal{P}$.

Algorithm 4.1 For an arbitrary initial point $x_{0}$, sequence $\left\{x_{n}\right\}$ is generated by the iteration:

$$
x_{n+1}=\left(1-\beta_{n}\right) x_{n}+\beta_{n} P_{C}\left[\left(1-\alpha_{n}\right)\left(I-\gamma P^{*} G^{*} G P\right)\right] x_{n},
$$

where $\alpha_{n}>0$ is a sequence in $(0,1)$ such that

(i) $\lim _{n \rightarrow \infty} \alpha_{n}=0, \sum_{n=0}^{\infty} \alpha_{n}=\infty$;

(ii) $\lim _{n \rightarrow \infty}\left|\alpha_{n+1}-\alpha_{n}\right|=0$;

(iii) $0<\liminf _{n \rightarrow \infty} \beta_{n} \leq \limsup _{n \rightarrow \infty} \beta_{n}<1$.

Lemma 4.2 If $z \in \operatorname{Fix}(T)=\operatorname{Fix}\left(I-\gamma P^{*} G^{*} G P\right)$, then for any $x$ we have $\|T x-z\|^{2} \leq \| x-$ $z\left\|^{2}-\beta(1-\beta)\right\| V x-x \|^{2}$, where $\beta$ and $V$ are the same in Lemma 2.5(1).

Proof By Lemma 2.5(1), we know that $T=\beta I+(1-\beta) V$, where $0<\beta<1$ and $V$ is a nonexpansive. It is clear that $z \in \operatorname{Fix}(T)=\operatorname{Fix}(V)$, and

$$
\begin{aligned}
\|T x-z\|^{2} & =\|\beta x+(1-\beta) V x-z\|^{2} \\
& \leq \beta\|x-z\|^{2}+(1-\beta)\|V x-z\|^{2}-\beta(1-\beta)\|V x-x\|^{2} \\
& \leq \beta\|x-z\|^{2}+(1-\beta)\|x-z\|^{2}-\beta(1-\beta)\|V x-x\|^{2} \\
& =\|x-z\|^{2}-\beta(1-\beta)\|V x-x\|^{2} .
\end{aligned}
$$


Theorem 4.3 The sequence $\left\{x_{n}\right\}$ generated by algorithm (4.1) converges strongly to a solution of the problem $\mathcal{P}$.

Proof For any solution $\hat{x}$ of the problem $\mathcal{P}$, according to Lemma 2.5, $\hat{x} \in \operatorname{Fix}\left(P_{C} T\right)=$ $\operatorname{Fix}\left(P_{C}\right) \cap \operatorname{Fix}(T)$, where $T=I-\gamma P^{*} G^{*} G P$, and

$$
\begin{aligned}
\left\|x_{n+1}-\hat{x}\right\|= & \left\|\left(1-\beta_{n}\right) x_{n}+\beta_{n} P_{C}\left[\left(1-\alpha_{n}\right) T\right] x_{n}-\hat{x}\right\| \\
= & \left\|\left(1-\beta_{n}\right)\left(x_{n}-\hat{x}\right)+\beta_{n}\left(P_{C}\left[\left(1-\alpha_{n}\right) T\right] x_{n}-\hat{x}\right)\right\| \\
\leq & \left(1-\beta_{n}\right)\left\|x_{n}-\hat{x}\right\|+\beta_{n}\left\|P_{C}\left[\left(1-\alpha_{n}\right) T\right] x_{n}-\hat{x}\right\| \\
\leq & \left(1-\beta_{n}\right)\left\|x_{n}-\hat{x}\right\| \\
& +\beta_{n}\left\|P_{C}\left[\left(1-\alpha_{n}\right) T\right] x_{n}-P_{C}\left[\left(1-\alpha_{n}\right) T\right] \hat{x}\right\| \\
& +\beta_{n}\left\|P_{C}\left[\left(1-\alpha_{n}\right) T\right] \hat{x}-\hat{x}\right\| \\
\leq & \left(1-\beta_{n}\right)\left\|x_{n}-\hat{x}\right\|+\beta_{n}\left(1-\alpha_{n}\right)\left\|x_{n}-\hat{x}\right\|+\beta_{n} \alpha_{n}\|\hat{x}\| \\
= & \left(1-\beta_{n} \alpha_{n}\right)\left\|x_{n}-\hat{x}\right\|+\beta_{n} \alpha_{n}\|\hat{x}\| \\
\leq & \max \left\{\left\|x_{n}-\hat{x}\right\|,\|\hat{x}\|\right\} .
\end{aligned}
$$

One can deduce that

$$
\left\|x_{n}-\hat{x}\right\| \leq \max \left\{\left\|x_{0}-\hat{x}\right\|,\|\hat{x}\|\right\}
$$

Hence, $\left\{x_{n}\right\}$ is bounded and so is $\left\{T x_{n}\right\}$. Moreover,

$$
\begin{aligned}
\left\|P_{C}\left[\left(1-\alpha_{n}\right) T\right] x_{n}-\hat{x}\right\| & \leq\left\|\left(1-\alpha_{n}\right) T x_{n}-\hat{x}\right\| \\
& =\left\|\left(1-\alpha_{n}\right)\left[T x_{n}-\hat{x}\right]-\alpha_{n} \hat{x}\right\| \\
& \leq\left(1-\alpha_{n}\right)\left\|x_{n}-\hat{x}\right\|+\alpha_{n}\|\hat{x}\| \\
& \leq \max \left\{\left\|x_{n}-\hat{x}\right\|,\|\hat{x}\|\right\} .
\end{aligned}
$$

Since $\left\{x_{n}\right\}$ is bounded, we see that $\left\{T x_{n}\right\},\left(1-\alpha_{n}\right) T x_{n}$, and $\left\{P_{C}\left[\left(1-\alpha_{n}\right) T\right] x_{n}\right\}$ are also bounded.

Let $z_{n}=P_{C}\left[\left(1-\alpha_{n}\right) T\right] x_{n}$, and $M>0$ such that $M=\sup _{n \geq 1}\left\{T x_{n}\right\}$. Noting that

$$
\begin{aligned}
\left\|P_{C}\left[\left(1-\alpha_{n+1}\right) T\right] x_{n}-P_{C}\left[\left(1-\alpha_{n}\right) T\right] x_{n}\right\| & \leq\left\|\left(1-\alpha_{n+1}\right) T x_{n}-\left(1-\alpha_{n}\right) T x_{n}\right\| \\
& =\left\|\left(\alpha_{n}-\alpha_{n+1}\right) T x_{n}\right\| \\
& \leq M\left|\alpha_{n}-\alpha_{n+1}\right| .
\end{aligned}
$$

One concludes that

$$
\begin{aligned}
\left\|z_{n+1}-z_{n}\right\|= & \left\|P_{C}\left[\left(1-\alpha_{n+1}\right) T\right] x_{n+1}-P_{C}\left[\left(1-\alpha_{n}\right) T\right] x_{n}\right\| \\
\leq & \left\|P_{C}\left[\left(1-\alpha_{n+1}\right) T\right] x_{n+1}-P_{C}\left[\left(1-\alpha_{n+1}\right) T\right] x_{n}\right\| \\
& +\left\|P_{C}\left[\left(1-\alpha_{n+1}\right) T\right] x_{n}-P_{C}\left[\left(1-\alpha_{n}\right) T\right] x_{n}\right\|
\end{aligned}
$$




$$
\begin{aligned}
& \leq\left(1-\alpha_{n+1}\right)\left\|x_{n+1}-x_{n}\right\|+\left\|P_{C}\left[\left(1-\alpha_{n+1}\right) T\right] x_{n}-P_{C}\left[\left(1-\alpha_{n}\right) T\right] x_{n}\right\| \\
& \leq\left(1-\alpha_{n+1}\right)\left\|x_{n+1}-x_{n}\right\|+M\left|\alpha_{n}-\alpha_{n+1}\right| .
\end{aligned}
$$

Since $0<\alpha_{n}<1$ and $\lim _{n \rightarrow \infty}\left|\alpha_{n+1}-\alpha_{n}\right|=0$, we have

$$
\left\|z_{n+1}-z_{n}\right\|-\left\|x_{n+1}-x_{n}\right\| \leq M\left|\alpha_{n}-\alpha_{n+1}\right|
$$

and

$$
\limsup _{n \rightarrow \infty}\left\|z_{n+1}-z_{n}\right\|-\left\|x_{n+1}-x_{n}\right\| \leq 0
$$

Applying Lemma 1.3, we get

$$
\lim _{n \rightarrow \infty}\left\|P_{C}\left[\left(1-\alpha_{n}\right) T\right] x_{n}-x_{n}\right\|=\lim _{n \rightarrow \infty}\left\|z_{n}-x_{n}\right\|=0 .
$$

Hence,

$$
\begin{aligned}
\left\|x_{n+1}-x_{n}\right\| & =\left\|\left(1-\beta_{n}\right) x_{n}+\beta_{n} P_{C}\left[\left(1-\alpha_{n}\right) T\right] x_{n}-x_{n}\right\| \\
& =\beta_{n}\left\|P_{C}\left[\left(1-\alpha_{n}\right) T\right] x_{n}-x_{n}\right\| \rightarrow 0 .
\end{aligned}
$$

Let $R_{n}$ and $R$ be defined by

$$
\begin{aligned}
& R_{n} x:=P_{C}\left\{\left(1-\alpha_{n}\right)\left[I-\gamma P^{*} G^{*} G P\right]\right\} x=P_{C}\left[\left(1-\alpha_{n}\right) T x\right], \\
& R x:=P_{C}\left(I-\gamma P^{*} G^{*} G P\right) x=P_{C}(T x) .
\end{aligned}
$$

Noting that

$$
\begin{aligned}
\left\|x_{n}-R x_{n}\right\| & \leq\left\|x_{n}-x_{n+1}\right\|+\left\|x_{n+1}-R x_{n}\right\| \\
& =\left\|x_{n}-x_{n+1}\right\|+\left\|\left(1-\beta_{n}\right) x_{n}+\beta_{n} R_{n} x_{n}-R x_{n}\right\| \\
& \leq\left\|x_{n}-x_{n+1}\right\|+\left(1-\beta_{n}\right)\left\|x_{n}-R x_{n}\right\|+\beta_{n}\left\|R_{n} x_{n}-R x_{n}\right\| .
\end{aligned}
$$

So, we have

$$
\begin{aligned}
\left\|x_{n}-R x_{n}\right\| & \leq\left\|x_{n}-x_{n+1}\right\| / \beta_{n}+\left\|R_{n} x_{n}-R x_{n}\right\| \\
& =\left\|x_{n}-x_{n+1}\right\| / \beta_{n}+\left\|P_{C}\left[\left(1-\alpha_{n}\right) T\right] x_{n}-P_{C} T x_{n}\right\| \\
& \leq\left\|x_{n}-x_{n+1}\right\| / \beta_{n}+\left\|\left(1-\alpha_{n}\right) T x_{n}-T x_{n}\right\| \\
& \leq\left\|x_{n}-x_{n+1}\right\| / \beta_{n}+M \alpha_{n} .
\end{aligned}
$$

By assumption, we have

$$
\lim _{n \rightarrow \infty}\left\|x_{n}-R x_{n}\right\|=0 .
$$

Furthermore, $\left\{x_{n}\right\}$ is bounded, there exists a subsequence of $\left\{x_{n}\right\}$ which converges weakly to a point $\check{x}$. Without loss of generality, we may assume that $\left\{x_{n}\right\}$ converges weakly to $\check{x}$. 
Since $\left\|R x_{n}-x_{n}\right\| \rightarrow 0$, using the demiclosedness principle we know that $\check{x} \in \operatorname{Fix}(R)=$ $\operatorname{Fix}\left(P_{C} T\right)=\operatorname{Fix}\left(P_{C}\right) \cap \operatorname{Fix}(T)=\Gamma$.

Finally, we will prove that $\lim _{n}\left\|x_{n+1}-\check{x}\right\|=0$. In fact,

$$
\begin{aligned}
\left\|x_{n+1}-\check{x}\right\|^{2}= & \left\|\left(1-\beta_{n}\right) x_{n}+\beta_{n} P_{C}\left[\left(1-\alpha_{n}\right) T\right] x_{n}-P_{C} T \check{x}\right\|^{2} \\
\leq & \left(1-\beta_{n}\right)\left\|x_{n}-\check{x}\right\|^{2}+\beta_{n}\left\|P_{C}\left[\left(1-\alpha_{n}\right) T\right] x_{n}-P_{C} T \check{x}\right\|^{2} \\
\leq & \left(1-\beta_{n}\right)\left\|x_{n}-\check{x}\right\|^{2}+\beta_{n}\left\|\left(1-\alpha_{n}\right) T x_{n}-\check{x}\right\|^{2} \\
= & \left(1-\beta_{n}\right)\left\|x_{n}-\check{x}\right\|^{2}+\beta_{n}\left\|\left(1-\alpha_{n}\right)\left(T x_{n}-\check{x}\right)+\alpha_{n} \check{x}\right\|^{2} \\
= & \left(1-\beta_{n}\right)\left\|x_{n}-\check{x}\right\|^{2}+\beta_{n}\left[\left(1-\alpha_{n}\right)^{2}\left\|T x_{n}-\check{x}\right\|^{2}+\alpha_{n}^{2}\|\check{x}\|^{2}\right. \\
& \left.+2 \alpha_{n}\left(1-\alpha_{n}\right)\left\langle T x_{n}-\check{x},-\check{x}\right\rangle\right] \\
\leq & \left(1-\beta_{n}\right)\left\|x_{n}-\check{x}\right\|^{2}+\beta_{n}\left[\left(1-\alpha_{n}\right)\left\|x_{n}-\check{x}\right\|^{2}+\alpha_{n}^{2}\|\check{x}\|^{2}\right. \\
& \left.+2 \alpha_{n}\left(1-\alpha_{n}\right)\left\langle T x_{n}-\check{x},-\check{x}\right\rangle\right] \\
= & \left(1-\alpha_{n} \beta_{n}\right)\left\|x_{n}-\check{x}\right\|^{2}+\alpha_{n} \beta_{n}\left[2\left(1-\alpha_{n}\right)\left\langle T x_{n}-\check{x},-\check{x}\right\rangle+\alpha_{n}\|\check{x}\|^{2}\right] .
\end{aligned}
$$

Using Lemma 1.2, we only need to prove that

$$
\limsup _{n \rightarrow \infty}\left\langle T x_{n}-\check{x},-\check{x}\right\rangle \leq 0
$$

Applying Lemma 2.5, $T$ is averaged, that is $T=\beta I+(1-\beta) V$, where $0<\beta<1$ and $V$ is nonexpansive. Hence, for $z \in \operatorname{Fix}\left(P_{C} T\right)$, we have

$$
\begin{aligned}
\left\|x_{n+1}-z\right\|^{2} & =\left\|\left(1-\beta_{n}\right) x_{n}+\beta_{n} P_{C}\left[\left(1-\alpha_{n}\right) T\right] x_{n}-z\right\|^{2} \\
& \leq\left(1-\beta_{n}\right)\left\|x_{n}-z\right\|^{2}+\beta_{n}\left\|\left(1-\alpha_{n}\right) T x_{n}-z\right\|^{2} \\
& =\left(1-\beta_{n}\right)\left\|x_{n}-z\right\|^{2}+\beta_{n}\left\|\left(1-\alpha_{n}\right)\left(T x_{n}-z\right)-\alpha_{n} z\right\|^{2} \\
& \leq\left(1-\beta_{n}\right)\left\|x_{n}-z\right\|^{2}+\beta_{n}\left[\left(1-\alpha_{n}\right)\left\|T x_{n}-z\right\|^{2}+\alpha_{n}\|z\|^{2}\right] \\
& \leq\left(1-\beta_{n}\right)\left\|x_{n}-z\right\|^{2}+\beta_{n}\left[\left\|T x_{n}-z\right\|^{2}+\alpha_{n}\|z\|^{2}\right] .
\end{aligned}
$$

By Lemma 4.2, we have

$$
\begin{aligned}
\left\|x_{n+1}-z\right\|^{2} \leq & \left(1-\beta_{n}\right)\left\|x_{n}-z\right\|^{2} \\
& +\beta_{n}\left[\left\|x_{n}-z\right\|^{2}-\beta(1-\beta)\left\|V x_{n}-x_{n}\right\|^{2}+\alpha_{n}\|z\|^{2}\right] \\
\leq & \left\|x_{n}-z\right\|^{2}-\beta_{n} \beta(1-\beta)\left\|V x_{n}-x_{n}\right\|^{2}+\beta_{n} \alpha_{n}\|z\|^{2} .
\end{aligned}
$$

Let $N>0$ such that $\left\|x_{n}-z\right\| \leq N$ for all $n$, then it concludes that

$$
\begin{aligned}
\beta_{n} \beta(1-\beta)\left\|V x_{n}-x_{n}\right\|^{2} & \leq\left\|x_{n}-z\right\|^{2}-\left\|x_{n+1}-z\right\|^{2}+\beta_{n} \alpha_{n}\|z\|^{2} \\
& \leq 2 N\left|\left\|x_{n}-z\right\|-\left\|x_{n+1}-z\right\|\right|+\beta_{n} \alpha_{n}\|z\|^{2} \\
& \leq 2 N\left\|x_{n}-x_{n+1}\right\|+\beta_{n} \alpha_{n}\|z\|^{2} .
\end{aligned}
$$


Hence,

$$
\beta(1-\beta)\left\|V x_{n}-x_{n}\right\|^{2} \leq \frac{2 N\left\|x_{n}-x_{n+1}\right\|}{\beta_{n}}+\alpha_{n}\|z\|^{2} .
$$

Since $\left\|x_{n}-x_{n+1}\right\| \rightarrow 0$, we get

$$
\left\|V x_{n}-x_{n}\right\| \rightarrow 0
$$

Therefore,

$$
\left\|T x_{n}-x_{n}\right\| \rightarrow 0
$$

It follows that

$$
\limsup _{n \rightarrow \infty}\left\langle T x_{n}-\check{x},-\check{x}\right\rangle=\limsup _{n \rightarrow \infty}\left\langle x_{n}-\check{x},-\check{x}\right\rangle .
$$

Since $\left\{x_{n}\right\}$ converges weakly to $\check{x}$, it follows that

$$
\limsup _{n \rightarrow \infty}\left\langle T x_{n}-\check{x},-\check{x}\right\rangle \leq 0 \text {. }
$$

Similar to the proof of Theorem 4.3, we can get the result that the following iterative algorithm converges strongly to a solution of the problem $\mathcal{P}$ also. Since the proof is similar to Theorem 4.3, we omit it.

Algorithm 4.4 For an arbitrary initial point $x_{0}$, sequence $\left\{x_{n}\right\}$ is generated by the iteration:

$$
x_{n+1}=\left(1-\beta_{n}\right)\left(1-\alpha_{n}\right)\left(I-\gamma P^{*} G^{*} G P\right) x_{n}+\beta_{n} P_{C}\left[\left(1-\alpha_{n}\right)\left(I-\gamma P^{*} G^{*} G P\right)\right] x_{n},
$$

where $\alpha_{n}>0$ is a sequence in $(0,1)$ such that

(i) $\lim _{n \rightarrow \infty} \alpha_{n}=0, \sum_{n=0}^{\infty} \alpha_{n}=\infty$;

(ii) $\lim _{n \rightarrow \infty}\left|\alpha_{n+1}-\alpha_{n}\right|=0$;

(iii) $0<\liminf _{n \rightarrow \infty} \beta_{n} \leq \lim \sup _{n \rightarrow \infty} \beta_{n}<1$.

\section{Competing interests}

The authors declare that they have no competing interests.

Authors' contributions

Recently, Eckstein and Svaiter present some splitting methods for finding a zero of the sum of monotone operator $A$ and $B$. However, the algorithms are largely dependent on the maximal monotonicity of $A$ and $B$. In this paper, we describe some algorithms for finding a zero of the sum of $A$ and $B$ which ignore the conditions of the maximal monotonicity of $A$ and $B$

\section{Author details}

${ }^{1}$ Department of Mathematics, Tianjin Polytechnic University, Tianjin, 300387, P.R. China. ${ }^{2}$ Tianjin Vocational Institute, Tianjin, 300410, P.R. China.

\section{Acknowledgements}

This research was supported by NSFC Grants No:1 1071279; No:11226125; No:11301379. 


\section{References}

1. Eckstein, J, Svaiter, BF: A family of projective splitting methods for the sum of two maximal monotone operators. Math. Program. 111, 173-1199 (2008)

2. Eckstein, J, Bertsekas, D: On the Douglas-Ratford splitting method and the proximal point algorithm for maximal monotone operators. Math. Program. 55, 293-318 (1992)

3. Lions, PL: Une méthode itérative de résolution d'une inéquation variationelle. Isr. J. Math. 31، 204-208 (1978)

4. Passty, GB: Ergodic convergence to a zero of the sum of monotone operators in Hilbert space. J. Math. Anal. Appl. 72, 383-390 (1979)

5. Tseng, P: A modified forward-backward splitting method for maximal monotone mappings. SIAM J. Control Optim. 38, 431-446 (2000)

6. Geobel, K, Kirk, WA: Topics in Metric Fixed Point Theory. Cambridge Studies in Advanced Mathematics, vol. 28. Cambridge University Press, Cambridge (1990)

7. Geobel, K, Reich, S: Uniform Convexity, Nonexpansive Mappings, and Hyperbolic Geometry. Dekker, New York (1984)

8. Aoyama, K, Kimura, Y, Takahashi, W, Toyoda, M: Approximation of common fixed points of a countable family of nonexpansive mappings in a Banach space. Nonlinear Anal., Theory Methods Appl. 67(8), 2350-2360 (2007)

9. Suzuki, T: Strong convergence theorems for infinite families of nonexpansive mappings in general Banach space. Fixed Point Theory Appl. 1, 103-123 (2005)

10. Engl, HW, Hanke, M, Neubauer, A: Regularization of Inverse Problems. Mathematics and Its Applications, vol. 375. Kluwer Academic, Dordrecht (1996)

11. Byrne, C: A unified treatment of some iterative algorithms in signal processing and image reconstruction. Inverse Probl. 20(1), 103-120 (2004)

10.1186/1029-242X-2014-349

Cite this article as: Shi et al.: Iterative algorithms for finding the zeroes of sums of operators. Journal of Inequalities and Applications 2014, 2014:349

\section{Submit your manuscript to a SpringerOpen ${ }^{\circ}$ journal and benefit from:}

- Convenient online submission

- Rigorous peer review

- Immediate publication on acceptance

- Open access: articles freely available online

- High visibility within the field

- Retaining the copyright to your article 